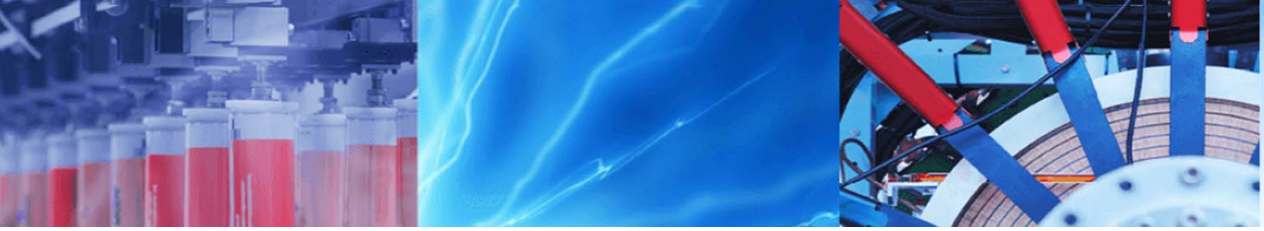

Research Article

\title{
Evaluation of structural, Hirshfeld surface and density functional theory of novel NLO thienyl chalcone single crystal
}

\author{
C. Shruthi ${ }^{1} \cdot$ V. Ravindrachary ${ }^{1}$ (D) B. Guruswamy ${ }^{1} \cdot$ D. Jagadeesh Prasad ${ }^{2} \cdot$ Janet Goveas $^{2}$
}

Received: 26 July 2019 / Accepted: 27 September 2019 / Published online: 27 November 2019

(c) Springer Nature Switzerland AG 2019

\begin{abstract}
A novel NLO-active chalcone derivative (E)-1-(5-chlorothiophene-2-yl)-3-(4-dimethylamino) phenyl) prop-2-en-1-one has been synthesized by adopting Claisen-Schmidt condensation reaction method. Using DMF as solvent, single crystals of the compound were grown by solution growth technique. The grown crystals are of diamond-shaped dark red colour in nature, and these crystals are characterized using different experimental methods. NMR and LC-MS spectral methods are used to confirm the molecular structure of the synthesized compound. The observed fluorescence spectrum clearly signifies that the newly grown crystal could be utilized as a new green light-emitting material. The molecular 3-dimensional hirshfeld surface and the corresponding 2-dimensional finger print plot representation quantifying the percentage contribution of each individual atom present in the compound were studied. From the density functional theory, the values of HOMO, LUMO and their energy gap were determined. The values of global chemical reactivity descriptors such as chemical hardness $(\eta)$, global softness $(\sigma)$, electrophilicity $(\omega)$, ionization potential $(I)$ and chemical potential $(\mu)$ have been calculated. The molecular electrostatic potential map identifies the potential sites for electrophilic and nucleophilic attack in the molecule. The NLO parameters such as molecular polarizability $(\alpha)$, dipole moment $(\mu)$ and first-order hyperpolarizability $(\beta)$ were determined using density functional theory.
\end{abstract}

Keywords Thienyl chalcone $\cdot$ Hirshfeld surface $\cdot$ DFT $\cdot$ First-order hyperpolarizability

\section{Introduction}

The organic materials with superior nonlinear optical (NLO) properties have grabbed the attention of researchers in view of their enchanting applications in the fields like optoelectronics and photonics such as optical computing, frequency shifting and optical information processing [1]. Many organic, inorganic and molecular crystalline materials have been recognized as an effective NLO material. The development of organic materials particularly for NLO applications in recent times has turned out to be an active domain of research in the realm of science and technology due to their effortless synthesis process and greater NLO efficiency that has a huge impact on technological applications. But the large molecular hyperpolarizability in organic materials exhibits a fascinating NLO effects which in turn attracts the researchers in developing these materials to a greater extent. The main reason behind the widespread of organic materials is the availability of molecular charge transfer capability to a greater extent which in turn is responsible for the achievement of vast nonlinear optical response. In general, the structure of the organic NLO materials with donor-acceptor (D-A) $\pi$-electron conjugated systems which induce an asymmetric electronic distribution that leads to a greater mobility of an electron density which further enhances the optical nonlinearity in the material. Also, the organic NLO materials retain high degree of delocalization as it is composed of week

V. Ravindrachary, vravi2000@yahoo.com | 'Department of Physics, Mangalore University, Mangalagangothri 574199, India. ${ }^{2}$ Department of Chemistry, Mangalore University, Mangalagangothri 574199, India. 
van der Waals forces and hydrogen bonds. As a result, the sliding of delocalized $\pi$ electrons between the donor and acceptor groups on either ends of the molecule results in the increased optical nonlinearity of the material [2]. Another interesting feature of organic materials for their widespread utility is its structural modification. One can easily modify the structure of these materials according to the required design principle by substituting the different functional groups to procure the desired optical property. Therefore, an additional attention has been shown towards the synthesis of organic materials [3]. Though it has been remarkably studied over the several decades, the organic materials exhibits few major drawbacks such as poor thermal and mechanical stability, absence of robustness, etc. which strictly obstructs their use in practical applications. Also it is quite difficult to grow them as large-size single crystals [4].

Chalcones ( $\alpha, \beta$-unsaturated ketone) are the most special class of organic cross-conjugated NLO chromophore that are very much attractive and found to be the most potential candidate for NLO studies owing to their outstanding features like good crystallizability, greater NLO efficiency, huge transparency, chemical inertness, phase matching properties, excellent blue light transmittance and high laser damage threshold [5]. Chalcone derivatives are more preferably studied for NLO applications because of the existence of conjugated $\pi$ electrons in them which in turn is responsible for the production of greater secondorder nonlinear coefficients which are very much useful for applications like optical parametric amplification and SHG [6].

In present days, the prediction of physical and chemical properties of the molecular structures through the advanced quantum chemical approach using computational techniques is playing an outstanding role by disclosing the valuable applications of organic materials in the field of nonlinear optics [7]. This is mainly due to the fact that, while choosing the material for industrial application such as photonics, the physical properties of the crystal play an important role. Therefore, it is necessary to optimize the crystal properties for the industrial applications as well as for the technological applications. Among the various computational techniques available for the successive evaluation of NLO parameters, the density functional theoretical (DFT) method has proven to be the most effective and suitable tool for optimizing the structures for varied applications [6]. Hence, the present work sheds light on the evaluation of NLO parameters through DFT method and the values of dipole moment $(\mu)$, polarizability $(\alpha)$ and the first-order molecular hyperpolarizability $(\beta)$ have been calculated.

\section{Experimental}

\subsection{Synthesis of the compound}

The compound (E)-1-(5-chlorothiophene-2-yl)-3-(4dimethylamino)phenyl)prop-2-en-1-one was synthesized using standard Claisen-Schmidt condensation method [3]. For the present synthesis, an equal ratio of 2-acetyl-5chloro thiophene $(0.01 \mathrm{~mol})$ and 4-dimethylaminobenzaIdehyde $(0.01 \mathrm{~mol})$ compounds was used which are purchased from Sigma-Aldrich chemicals Pvt Ltd., Bangalore. Molecular structure of the compound was confirmed using $400 \mathrm{MHz}{ }^{1} \mathrm{H}-\mathrm{NMR}$ spectrum. The fluorescence spectrum of the present compound was carried out using HITACHIF-7000 fluorescence spectrophotometer in solution form using ethanol as solvent in the wavelength ranging between 200 and $800 \mathrm{~nm}$. The single crystals of the compound were grown using solution growth technique with DMF as solvent. Molecular structure and single crystal of the compound are shown in Fig. 1.

\subsection{LC-MS analysis}

The mass spectrum of the present compound is shown in Fig. 2. The observed spectra show a molecular ion peak at $\mathrm{m} / \mathrm{z} 292.09(\mathrm{M}+\mathrm{H})^{+}$corresponding to its molecular formula $\mathrm{C}_{15} \mathrm{H}_{14} \mathrm{CINOS}$ along with its isotopic peak at $\mathrm{m} / \mathrm{z}$ $294.08(\mathrm{M}+3 \mathrm{H})^{+}$which confirms the molecular structure of the compound.

\section{$2.3{ }^{1} \mathrm{H}-\mathrm{NMR}$ analysis}

The observed ${ }^{1} \mathrm{H}$-NMR spectrum of the present compound in DMSO solvent is shown in Fig. 3. Figure 3 shows a singlet at $\delta 2.97 \mathrm{ppm}$ integrated to the 6 protons of $\mathrm{N}-\left(\mathrm{CH}_{3}\right)_{2}$ group. Protons of thiophene ring resonated as 2 doublets at $\delta 7.29$ and $\delta 8.11 \mathrm{ppm}$, both with the same coupling constants of $J=4.4 \mathrm{~Hz}$. The 2 vinyl protons split each other into 2 doublets one centred at $\delta 7.50$ and the other at $\delta$ $7.62 \mathrm{ppm}$ with $J=15.6 \mathrm{~Hz}$, indicating trans proton-proton coupling across a double bond. The 2 doublets at $\delta$ $6.70 \mathrm{ppm}$ with $J=8.4 \mathrm{~Hz}$ and $\delta 7.66 \mathrm{ppm}$ with $J=8.8 \mathrm{~Hz}$ each integrated to the 2 protons of dimethylamino phenyl

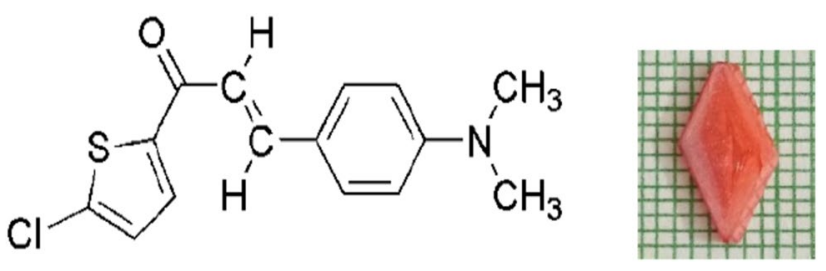

Fig. 1 As-grown crystal of the synthesized compound with molecular structure 
1800833-CDPP 3 (0.068) Cm (3:4)

TOF MS AP+

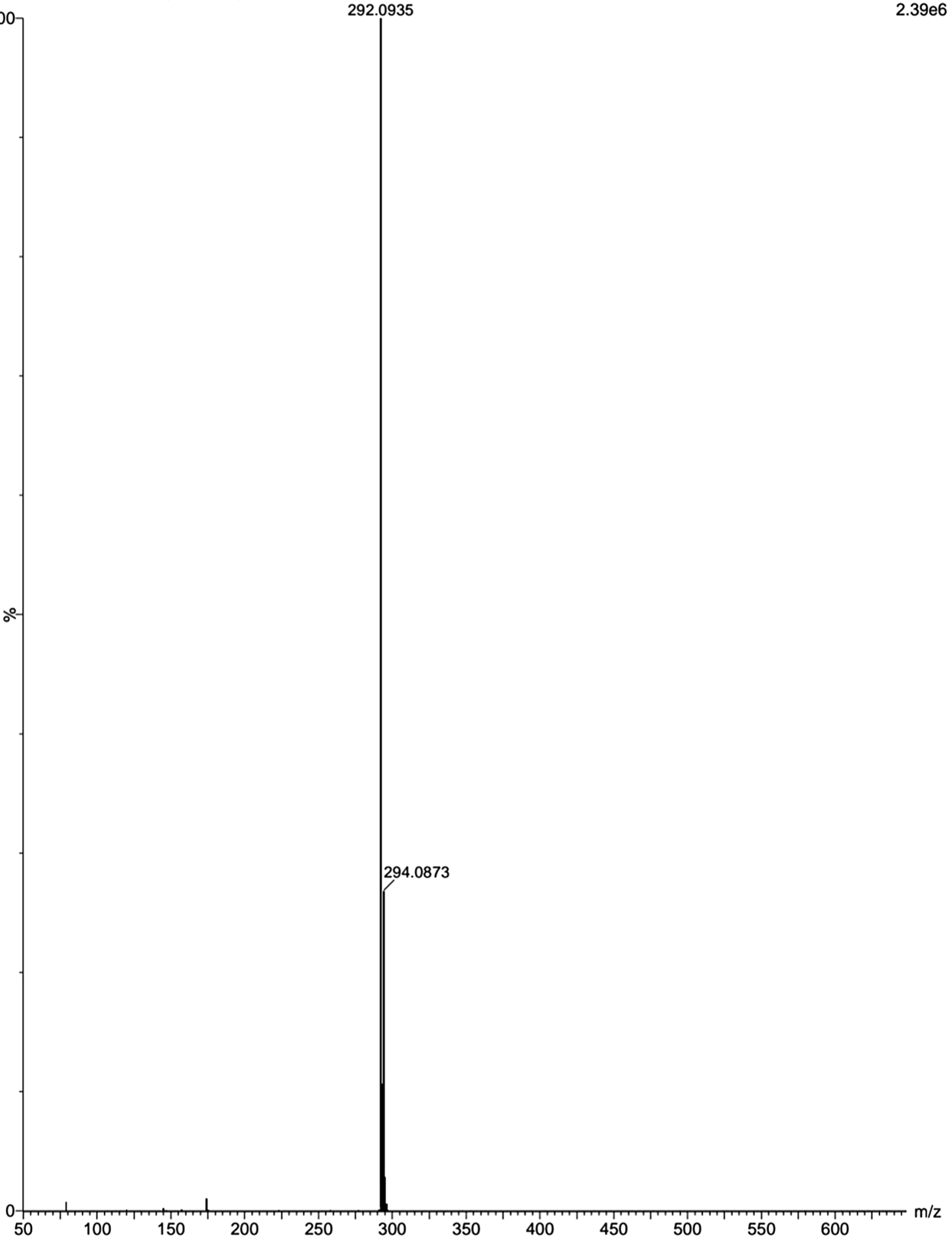

Fig. 2 LC-MS spectrum of the compound 


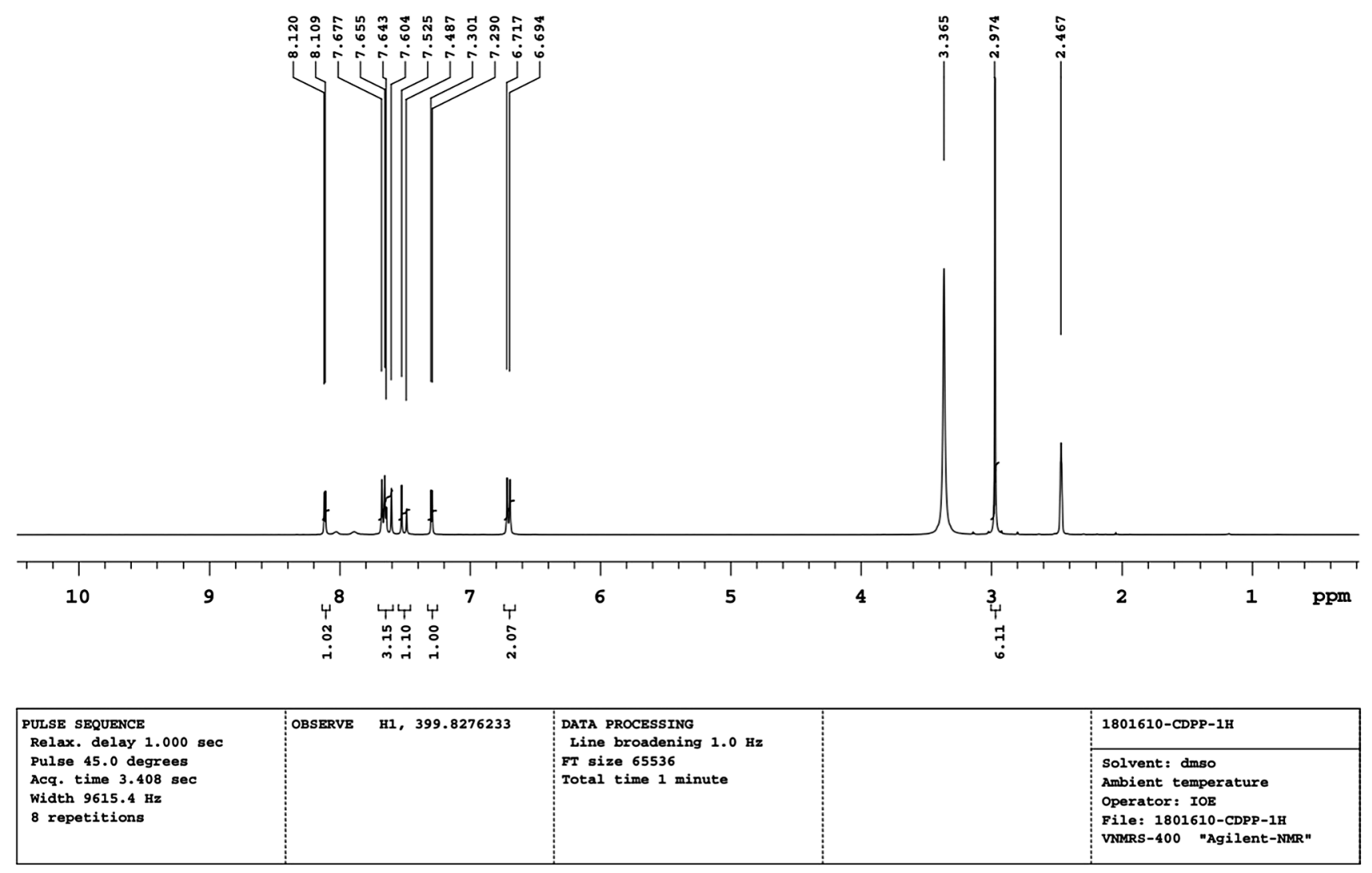

Fig. $3{ }^{1} \mathrm{H}-\mathrm{NMR}$ spectrum of the compound

moiety. These results confirm the molecular structure shown in Fig. 1.

\section{Results and discussion}

\subsection{Fluorescence analysis}

The fluorescence spectrum of the present compound is shown in Fig. 4. The figure shows a broad emission peak at $554 \mathrm{~nm}$ wavelength when the material is excited between the maximum wavelength $\left(\lambda_{\max }\right)$ of $400-450 \mathrm{~nm}$. (The value of $\lambda_{\max }$ has been obtained from the UV-Vis analysis of the present chalcone molecule [8].) The observed emission at $554 \mathrm{~nm}$ in the spectrum clearly signifies the green emission property of the material that could be utilized for optical applications as new green light-emitting material [1].

\subsection{Hirshfeld surface (HS) analysis}

Hirshfeld surface analysis is a wonderful tool, which helps to understand the intermolecular interactions apart from their graphical visualization. Analysis and calculations of the Hirshfeld surface were carried out using the observed CIF file of single-crystal structure analysis, and the finger print plots were plotted using the software Crystal Explorer version 3.0. The $d_{\text {norm }}$ plots were mapped with colour scale in between -0.093 au (blue) and 1.432 au (red), respectively, which is shown in Fig. 5. The expanded 2D fingerprint plots (Fig. 6) [9] were displayed in the range of 0.6-2.8 $\AA$ with the $d_{\mathrm{e}}$ and $d_{\mathrm{i}}$ distance scales displayed on the graph axes. $d_{\mathrm{i}}$ is the closest internal distance from a given point on the Hirshfeld surface, and $d_{\mathrm{e}}$ is the closest external contact.

The fingerprint plots reveal the percentage contribution of intermolecular contacts to the surface which can be represented in terms of colour codes. The $\mathrm{H}-\mathrm{H}$ (36.3\%) contact has the maximum contribution among others. The $\mathrm{C}-\mathrm{H}(16.0 \%), \mathrm{Cl}-\mathrm{H}(14.7 \%), \mathrm{O}-\mathrm{H}(10.7 \%), \mathrm{S}-\mathrm{H}(8.9 \%), \mathrm{C}-\mathrm{C}$ (6.0\%), $\mathrm{C}-\mathrm{Cl}(3.7 \%)$ and $\mathrm{C}-\mathrm{S}(2.5 \%)$ contacts contribute to the total area of the surface as shown in Fig. 6 . These contacts are highlighted on the molecular surface using conventional mapping of $d_{\text {norm }}$, shape index and curvedness as shown in Fig. 5. The regions with red and blue colour represent the shorter and longer inter-contacts, respectively [10]. 


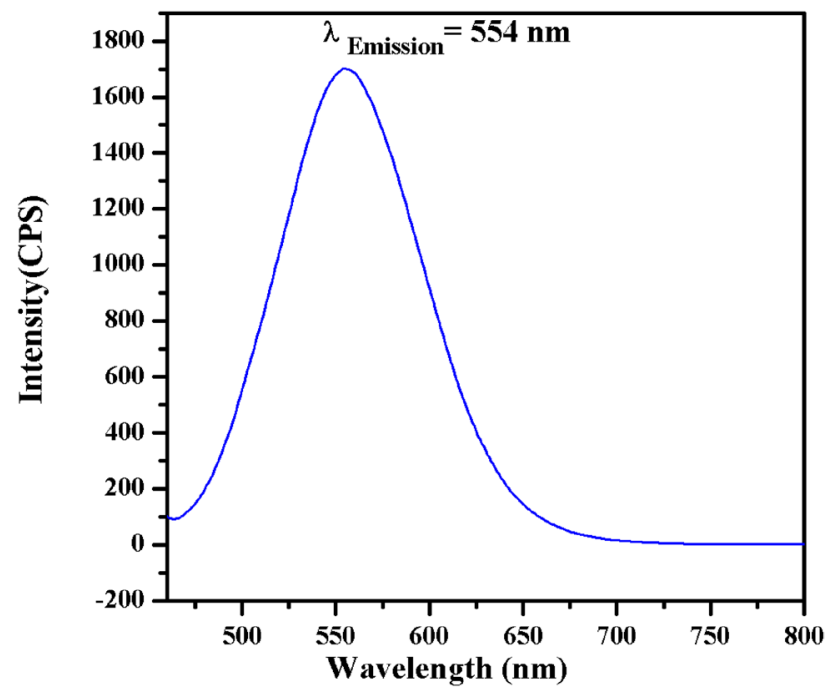

Fig. 4 Fluorescent spectrum of the grown crystal

\subsection{DFT calculations}

\subsubsection{HOMO-LUMO analysis}

The interaction exhibited by molecule with other species in the crystal structure can be well predicted with the aid of frontier molecular orbitals (FMO), namely HOMO and LUMO. Highest occupied molecular orbital (HOMO), the outermost orbital, acts as an electron donor as it contains electrons, whereas the lowest unoccupied molecular orbital (LUMO) accepts electrons as an electron acceptor [11]. The energy gap between these two orbitals clearly explains the kinetic stability, chemical reactivity, charge transfer interaction and some optical properties of the molecule. Red and green colour in Fig. 7 represents the positive and negative phases of the wave functions, respectively [12]. The small value of energy gap is connected with the significant intramolecular charge transfer of the molecule from donor group to an acceptor group along the path of $\pi$-conjugation. On the other hand, the greater energy gap value represents the greater stability of the molecule but relatively shows lower chemical reactivity. The molecule with greater $\mathrm{E}_{\mathrm{HOMO}}$ value is good electron donors, whereas the molecule with smaller $\mathrm{E}_{\mathrm{LUMO}}$ values is good electron acceptors [13]. For the present molecule, the energy values were computed using Gaussian 9 software package with B3LYP level with $6-31 \mathrm{G}(d, p)$ basis set. The calculated energy gap value along with their $E_{\text {номо }}$ and $\mathrm{E}_{\mathrm{LUMO}}$ values is given in Table 1. The value of energy gap is observed to be small (3.278 eV) which signifies the softness nature, greater chemical reactivity and polarizability of the molecule [14].

\subsubsection{Global chemical reactivity descriptors (GCRD)}

In order to understand the relationship between the structure, stability and the chemical reactivity of the molecules, the GCRD parameters play a crucial role and also it is very much useful in the quantitative establishment of structure property, activity in addition with toxicity relationships [15]. In view of this, the computed energy values of HOMO and LUMO are further utilized for calculating some chemical properties of the molecule like chemical hardness $(\eta)$, global softness $(\sigma)$, electrophilicity $(\omega)$, electronegativity $(\chi)$ and chemical potential $(\mu)$. According to FMO theory, the energies of HOMO and LUMO are associated with the energies of ionization potential $(I)$ and electron affinity $(A)$. Therefore by considering the HOMO energy as I and LUMO energy as $A$, various GCRD parameters such as electronegativity $(\chi)=(I+A) / 2$, absolute hardness $(\eta)=(I-A) / 2$, global softness $(\sigma)=1 /(\eta)$, chemical potential $(\mu)=-(\chi)$ or $-(I+A) / 2$, electrophilicity index $(\omega)=\mu^{2} / 2 \eta$ have been calculated on the basis of Koopman's equations [16]. The estimated values are given in Table 1. The calculated values of GCRD parameters with the hardness value of $\eta=1.639 \mathrm{eV}$ indicate the greater chemical strength and stability of the present chalcone molecule.

\subsubsection{Molecular electrostatic potential (MEP)}

The MEP map provides the useful information regarding the reactive sites, delocalization, charge density, molecular size, shape and more significantly explains the positive, negative and neutral electrostatic potential in connection with different colour grading [17]. It is one of the powerful tools that foretells how the charge transfer interaction and the interaction between various kinds of geometries
Fig. $5 d_{\text {norm, }}$ shape index and curvedness mapped on Hirshfeld surface visualizing the molecular contacts

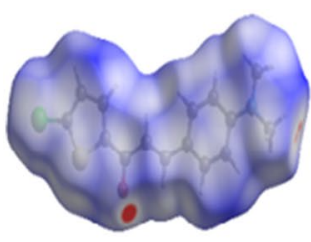

$\mathbf{d}_{\text {nom }}$

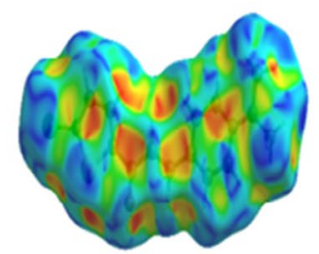

Shape index

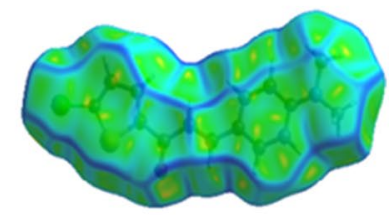

Curvedness 

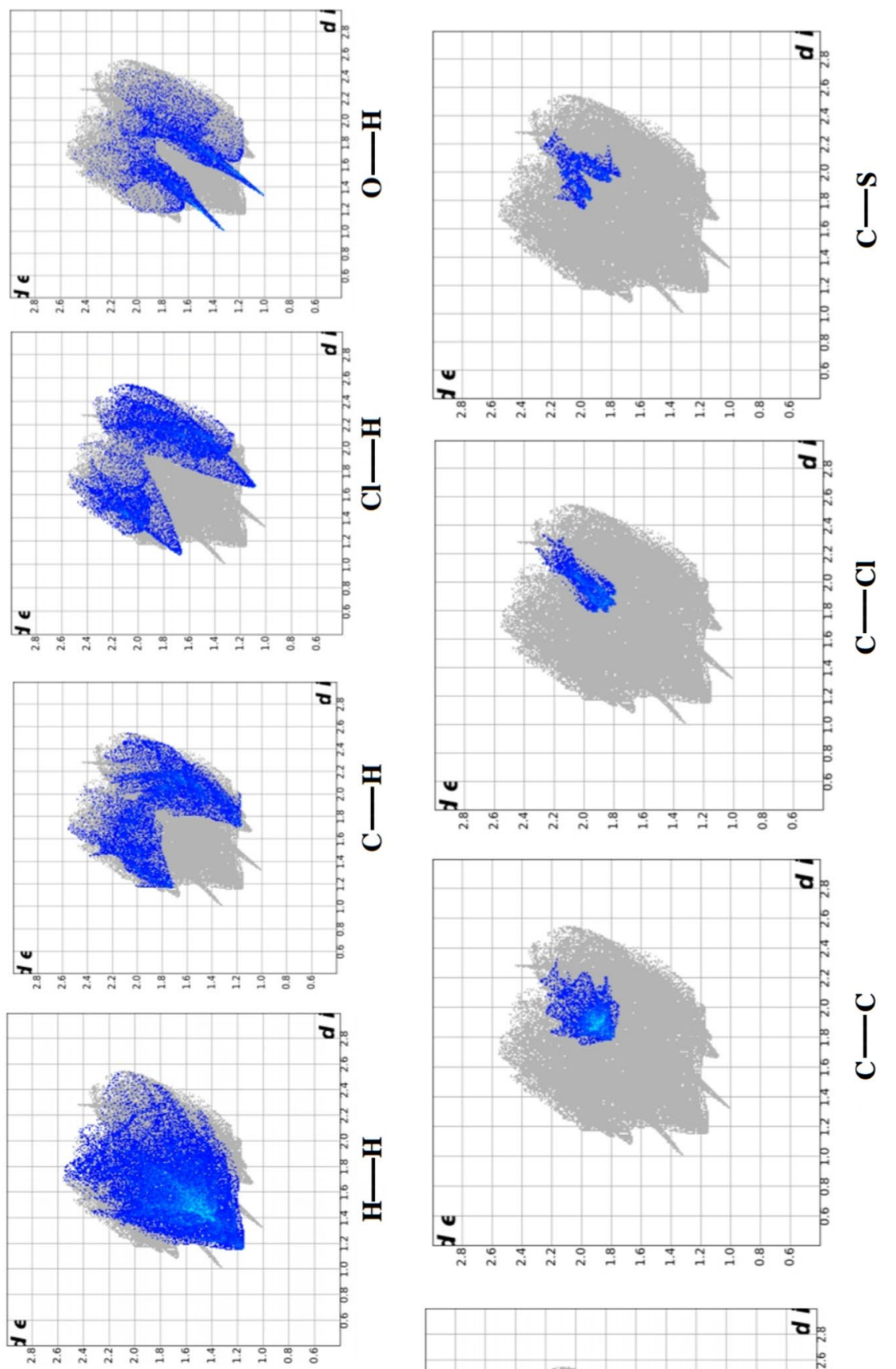


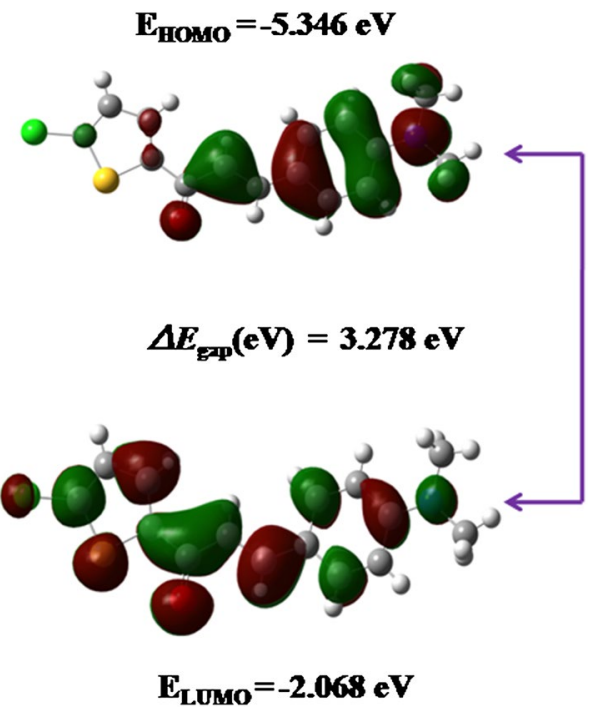

Fig. 7 HOMO-LUMO energy diagram of the compound

Table 1 Calculated electronic properties and quantum parameters of the present compound

\begin{tabular}{ll}
\hline Parameters & $\begin{array}{l}\text { Gas [DFT- } \\
\text { B3LYP/6-31 } \\
\mathrm{G}(d, p)]\end{array}$ \\
\hline$E_{\text {HOMO }}$ & $-5.346 \mathrm{eV}$ \\
$E_{\text {LUMO }}$ & $-2.068 \mathrm{eV}$ \\
$\Delta E_{\text {gap }}(\mathrm{eV})$ & $3.278 \mathrm{eV}$ \\
lonization potential $(I)$ & $5.346 \mathrm{eV}$ \\
Electron affinity $(A)$ & $2.068 \mathrm{eV}$ \\
Electronegativity $(\chi)$ & $3.707 \mathrm{eV}$ \\
Chemical hardness $(\eta)$ & $1.639 \mathrm{eV}$ \\
Global softness $(\sigma)$ & $0.6101 \mathrm{eV}-1$ \\
Electrophilicity $(\omega)$ & $4.19214 \mathrm{eV}$ \\
Chemical potential $(\mu)$ & $-3.707 \mathrm{eV}$ \\
\hline
\end{tabular}

could take place along with information on electrophilic, nucleophilic reactions and hydrogen bonding interactions. Red, blue and green are the 3 different colours on the surface that indicates the values of electrostatic potential. The blue and red colour on the surface represents the positive and negative values of the potential and is preferred sites for nucleophilic and electrophilic attacks, respectively. The zero potential will be indicated by green colour. The 3-dimensional MEP map of the chalcone molecule comprising various colour codes between deepest red and deepest blue, ranging from -6.613 to $6.613 \mathrm{e}^{-2}$, is shown in Fig. 8. From the figure, it is evident that the negative electrostatic potential (red and yellow region) is localized mainly on the carbonyl group of the molecule signifying $\mathrm{CO}$ as the preferable site for electrophilic attack which acts as an electron acceptor in the development of hydrogen bonds in crystal packing [18], whereas the positive electrostatic potential (light blue region) is located on dimethylamino phenyl moiety in the molecule which favours the nucleophilic attack.

\subsubsection{NLO analysis}

The relationship between the NLO response and the electronic structure of the system can be well described with the aid of quantum chemical approach. It is one of the most effective tools used for the investigation of NLO properties of the molecule and helps to determine the greater order hyperpolarizability of molecules in an inexpensive way for designing molecules by scrutinizing their potential before synthesis [1]. Here, the molecular properties such as dipole moment and polarizability tensors provide effective information regarding a charge distribution within a molecule. The polarizability tensor produced by an external electric field is the measure of electronic distribution in a molecule [11]. The evaluation of hyperpolarizability values is very much essential for the interpretation connecting molecular structure and the NLO property, particularly the second harmonic generation (SHG). The theoretical calculation of $\beta$ value signifies the direction of charge distribution that leads to second harmonics. For the present chalcone molecule, the calculated values of dipole moment $(\mu)$, total polarizability $(\alpha)$, anisotropy of polarizability $(\Delta \alpha)$ and first-order hyperpolarizability $(\beta)$ computed using DFT method with B3LYP level with 6-31 $\mathrm{G}(d, p)$ basis set are observed to be $9.4038 \mathrm{D}, 36.402 \times 10^{-24}$ esu, $51.25 \times 10^{-24}$ esu and $82.145 \times 10^{-30}$ esu, respectively, and are listed in Table 2 along with their tensor components obtained from the Gaussian frequency job output file. The values of $\mu, \alpha, \Delta a$ and $\beta$ are calculated using the following equations.

$$
\begin{aligned}
& \mu_{\text {tot }}=\left(\mu_{x}^{2}+\mu_{y}^{2}+\mu_{z}^{2}\right)^{\frac{1}{2}} \\
& \alpha_{\text {tot }}=\frac{1}{3}\left(\alpha_{x x}+\alpha_{y y}+\alpha_{z z}\right)
\end{aligned}
$$

$\Delta \alpha=\frac{1}{\sqrt{2}}\left[\left(\alpha_{x x}-\alpha_{y y}\right)^{2}+\left(\alpha_{y y}-\alpha_{z z}\right)^{2}+\left(\alpha_{z z}-\alpha_{x x}\right)^{2}+6 \alpha_{x z}^{2}+6 \alpha_{x y}^{2}+6 \alpha_{y z}^{2}\right]^{\frac{1}{2}}$ 


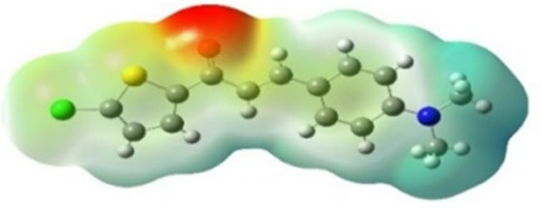

$-6.613 \mathrm{e}^{-2}$

$6.613 \mathrm{e}^{-2}$

Fig. 8 MEP of the chalcone molecule

Table 2 The electronic dipole moment ( $\mu$ ) (Debye), polarizability $(a)$ and first hyperpolarizability $(\beta)$ of the chalcone molecule

\begin{tabular}{llll}
\hline Parameter & a.u. & Parameter & a.u. \\
\hline$a_{x x}$ & 469.77 & $\beta_{x x x}$ & -9564.93 \\
$a_{x y}$ & 6.280 & $\beta_{x x y}$ & -2414.08 \\
$a_{y y}$ & 179.82 & $\beta_{x y y}$ & 265.69 \\
$a_{x z}$ & 1.987 & $\beta_{y y y}$ & 46.22 \\
$a_{y z}$ & -2.04 & $\beta_{x x z}$ & -39.79 \\
$a_{z z}$ & 87.31 & $\beta_{x y z}$ & -14.87 \\
$a_{\text {total }}$ & $36.402 \times 10^{-24} \mathrm{esu}$ & $\beta_{y y z}$ & -0.534 \\
$\Delta a$ & $51.251 \times 10^{-24} \mathrm{esu}$ & $\beta_{x z z}$ & 85.23 \\
& & $\beta_{y z z}$ & 20.90 \\
$\mu_{x}$ & 8.0888 & $\beta_{z z z}$ & 1.693 \\
$\mu_{y}$ & 4.7826 & $\beta_{\text {total }}$ & $82.145 \times 10^{-30} \mathrm{esu}$ \\
$\mu_{z}$ & 0.3605 & & \\
$\mu_{\text {total }}$ & $9.4038 \mathrm{D}$ & & \\
\hline
\end{tabular}

\section{Conclusions}

Single crystals of chalcone derivative with greater molecular hyperpolarizability have been carried out using solution growth technique, and their molecular structure was confirmed by ${ }^{1} \mathrm{H}-\mathrm{NMR}$ and LC-MS spectra. The fluorescence spectrum reveals the green emission property of the crystal. The inter-contacts involved in the crystal packing were visually analysed and quantized using Hirshfeld surface analysis as well as 2-dimensional finger print plot representation. The theoretical investigation carried out to estimate the energy values of HOMO, LUMO and the energy gap between them. The calculated energy gap value was observed to be low which indicates the softness of the molecule with more polarizability. High polarizability is an essential parameter for achieving greater nonlinear susceptibility. The calculated GCRD parameters such as ionization potential $(I)$, electron affinity $(A)$, electronegativity $(\chi)$, chemical hardness $(\eta)$, global softness $(\sigma)$, electrophilicity $(\omega)$ and chemical potential $(\mu)$ show that the material possesses good chemical strength and kinetic stability and hence favours the suitability of the material for optoelectronic applications. Molecular electrostatic potential (MEP) map predicts the suitable sites of electrophilic and nucleophilic attack in the molecule. The calculated NLO parameters such as molecular dipole moment $(\mu)$, mean polarizability $(\alpha)$, anisotropy of polarizability $(\Delta \alpha)$ and first-order hyperpolarizability $(\beta)$ are found to be greater than the standard urea which recommends the material as potential NLO material for future nonlinear applications.

$$
\beta_{\mathrm{tot}}=\left[\left(\beta_{X X X}+\beta_{X Y Z}+\beta_{X Z Z}\right)^{2}+\left(\beta_{Y Y Y}+\beta_{Y Z Z}+\beta_{Y X X}\right)^{2}+\left(\beta_{Z Z Z}+\beta_{Z X X}+\beta_{Z Y Y}\right)^{2}\right]^{\frac{1}{2}}
$$

The first-order molecular hyperpolarizability $\beta$ will be represented by a third rank tensor $3 \times 3 \times 3$ matrix. The 27 elements of the 3D matrix will be reduced to 10 components due to Kleinman symmetry. The calculated value of $\beta$ of the present chalcone molecule was observed to be greater than the urea $\left(0.13 \times 10^{-30}\right)$, which is an imperative prototypical molecule generally used as a threshold value for comparative purposes [14]. The greater value of $\beta$ could be attributed to the presence of intermolecular interactions such as $\mathrm{C}-\mathrm{H}-\mathrm{O}$ and $\pi . . . \pi$ in the molecule. The calculated values of $\mu, a, \Delta a$ and $\beta$ of the grown crystal suggest its suitability for NLO applications as the most potential and promising candidate.
Acknowledgements The authors wish to thank IOE, Vijnan Bhavan, University of Mysore, and USIC, Karnatak University, Dharwad, for instrumental facilities.

\section{Compliance with ethical standards}

Conflict of interest On behalf of all authors, the corresponding author states that there is no conflict of interest.

\section{References}

1. Dhamodharan P, Sathya K, Dhandapani M (2017) Systematic evaluation of a new organic material: 1 -methyl-1H-imidazol3-ium-2, 4, 6-trinitrobenzene-1, 3-bis (olate) for optoelectronics through spectral, structural, electrical, optical, quantum chemical and Hirshfeld surface studies. J Phys Chem Solids 104:175-184 
2. Rekha P, Peramaiyan G, NizamMohideen M, Mohan kumar R, Kanagadurai R (2016) Synthesis, growth and characterization of a new organic three dimensional framework: piperazin-1-ium 4-aminobenzenesulfonate. J Cryst Growth 441:18-25

3. Rajesh Kumar PC, Ravindrachary V, Janardhana K, Manjunath HR, Karegouda P, Crasta V, Sridhar MA (2011) Optical and structural properties of chalcone NLO single crystals. J Mol Struct 1005:1-7

4. Crasta V, Ravindrachary V, Lakshmi S, Pramod SN, Shridar MA, Shashidhara Prasad J (2005) Growth, characterization and crystal structure analysis of 1-(4-chlorophenyl)-3-(4-chlorophenyl)2-propen-1-one. J Cryst Growth 275:e329-e335

5. Prasad A, Muthu K, Meenatchi V, Rajasekar M, Agilandeshwari R, Meena K, Vijila Manonmoni J, Meenakshisundaram SP (2015) Optical, vibrational, NBO, first-order molecular hyperpolarizability and Hirshfeld surface analysis of a nonlinear optical chalcone. Spectrochim Acta Part A Mol Biomol Spectrosc 140:311-327

6. John JS, Sajan D, Narayana C, Joy N, Philip R (2018) Theoretical and experimental approach to the investigation of hyperpolarizability and charge transfer characteristics of NLO active 2', 3, 4, 4', 5-pentamethoxy chalcone with silver atoms adsorbed. Opt Mater 84:409-421

7. Chandra Shekhara Shetty T, Raghavendra S, Chidan Kumar CS, Naveen S, Maidur SR, Patil PS, Chandraju S, Ananthnag GS, Dharmaprakash SM (2018) Crystal structure, Hirshfeld and third-order nonlinear optical properties of 3-(4-dimethylamino) phenyl)-1-(4-methoxyphenyl) prop-2-en-1-one: a potential material for optical limiting applications. Opt Mater 86:138-147

8. Shruthi C, Ravindrachary V, Byrappa K, Guruswamy B, Jagadeesh Prasad D, Kumara K, Lokanath NK (2019) Growth and characterization of (E)-1-(5-Chlorothiophene-2-YI)-3-(4-Dimethylamino) Phenyl) Prop-2-En-1-One, novel NLO single crystal. Mater Sci Forum 962:3-9. https://doi.org/10.4028/www.scientific.net/ MSF.962.3

9. Seth SK (2013) Tuning the formation of MOFs by pH influence: $\mathrm{x}$-ray structural variations and Hirshfeld surface analyses of 2-amino-5-nitropyridine with cadmium chloride. Cryst Eng Commun 15:1772-1781

10. Kumara K, Shivalingegowda N, Mahadevaswamy LD, Kariyappa AK, Lokanath NK (2017) Crystal structure studies and Hirshfeld surface analysis of 5-(4-methoxyphenyl)-3-(thiophen-2-yl)4,5-dihydro-1H-pyrazole-1-carbothioamide. Chem Data Collect 9:251-262

11. Khanum H, Mashrai A, Siddiqui N, Ahmad M, Alam MJ, Ahmad S (2015) Structural elucidation, density functional calculations and contribution of intermolecular interactions in cholest-4-en-3-one crystals: insights from $x$-ray and Hirshfeld surface analysis. J Mol Struct 1084:274-283

12. Muthu K, Meenatchi V, Rajasekar M, Aditya Prasad A, Meena K, Agilandeshwari R, Kanagarajan V, Meenakshisundaram SP (2015) Combined theoretical and experimental studies on the molecular structure, spectral and Hirshfeld surface studies of NLO tris (thiourea) zinc(II) sulfate crystals. J Mol Struct 1091:210-221

13. Usman M, Arjmand F, Ahmad M, Khan MS, Ahmad I, Tabassum $S$ (2016) A comparative analyses of bioactive $\mathrm{Cu}(\mathrm{II})$ complexes using Hirshfeld surface and density functional theory (DFT) methods: DNA binding studies, cleavage and antibiofilm activities. Inorg Chim Acta 453:193-201

14. Thanigaimani K, Khalib NC, Temel E, Arshad S, Razak IA (2015) New supramolecular cocrystal of 2-amino-5-chloropyridine with 3-methylbenzoic acids: syntheses, structural characterization, Hirshfeld surfaces and quantum chemical investigations. J Mol Struct 1099:246-256

15. Shkir M, Patil PS, Arora M, AlFaify S, Algarni H (2017) An experimental and theoretical study on a novel donor- $\pi$-acceptor bridge type 2, 4, 5-trimethoxy-4'-chlorochalcone for optoelectronic applications: a dual approach. Spectrochim Acta Part A Mol Biomol Spectrosc 173:445-456

16. Kumara K, Dileep Kumar A, Naveen S, Ajay Kumar K, Lokanath NK (2018) Synthesis, spectral characterization and x-ray crystal structure studies of 3-(benzo [d][1, 3] dioxol-5-yl)-5-(3methylthiophen-2-yl)-4, 5-dihydro-1H-pyrazole-1-carboxamide: Hirshfeld surface, DFT and thermal analysis. J Mol Struct 1161(2018):285-298

17. Maidur SR, Patil PS, Ekbote A, Chia TS, Quah CK (2017) Molecular structure, second-and third-order nonlinear optical properties and DFT studies of a novel non-centrosymmetric chalcone derivative:(2E)-3-(4-fluorophenyl)-1-(4-\{[(1E)-(4-fluorophenyl) methylene] amino\} phenyl) prop-2-en-1-one. Spectrochim Acta Part A Mol Biomol Spectrosc 184:342-354

18. Ghanbari Niyaky S, Montazerozohori M, Masoudiasl A, White JM (2017) New five coordinated supramolecular structured cadmium complex as precursor for $\mathrm{CdO}$ nanoparticles: synthesis, crystal structure, theoretical and 3D Hirshfeld surface analyses. J Mol Struct 1131:201-211

Publisher's Note Springer Nature remains neutral with regard to jurisdictional claims in published maps and institutional affiliations. 